\title{
Tibia Malunion with Angular Deformity: Corrective Osteotomy and Intramedullary Fixation with the Chipping Technique
}

\author{
Nazri Mohd Yusof* and Muhammad Syukree Abot \\ Department of Orthopaedic, International Islamic University of Malaysia Medical Centre, Malaysia
}

Received: December 28, 2017; Published: January 04, 2018

*Corresponding author: Nazri Mohd Yusof, Department of Orthopaedic, Traumatology and Rehabilitation, Kulliyyah of Medicine, International Islamic University of Malaysia Medical Centre, Jalan Sultan Ahmad Shah, 25200 Kuantan, Pahang, Malaysia; Email: nazrimy2000@gmail.com

\begin{abstract}
There are few options for correcting severe malunion of tibial shaft. Acute correction by close wedge osteotomy followed by fixation with plate is a standard treatment for this but associated with shortening and non-union. Gradual correction with circular external fixation is able to correct the deformity without producing shortening. However it is uncomfortable and require tedious nursing and rehabilitative care. Chipping the non-union site was associated with a favorable outcome for femur fracture because it biologically enhances fracture healing. However, there is no report on chipping osteotomy for tibia mal-union. We consider chipping osteotomy as a treatment option for this patient who refuses to have external fixation for his treatment. Patient achieved corrected alignment of tibia and fracture union in 5 months. However he develops problem with wound healing of the wound.
\end{abstract}

\section{Case Report}

Mr NH is a 31-year old man who presented to us with a nineyear history of deformity of his right leg. He was involved in a road traffic accident in 2008 where he sustained an open fracture of his right tibia which was complicated with compartment syndrome. He underwent fasciotomy and external fixation of his right tibia. The fasciotomy wound later was closed by split skin grafting. Later the external fixator was removed and converted to plaster of Paris. However he then didn't turn up for follow up. Other than his bended leg, he claims that he was able to continue his work as a labourer with no limitation in his work and daily activity. He denied any history of fever, pain or sinus discharge from the leg. On examination, there was healed fasciotomy scar of the right leg on the medial and lateral surfaces of the leg with wasting of the calf muscle. There was also equinus deformity of the right ankle and clawed toes. This is evident by the formation of callosity along the metatarsal head at the plantar aspect.

There was also a clinically malunited fractures of the midshaft right tibia and fibula with an anterior angulation of the leg measuring 40 degrees on the goniometer. There was a limb length discrepancy of $0.5 \mathrm{~cm}$ on the right relative to the left. He had no distal neurovascular deficit (Figure 1A \& 1B). The range of motion of his right knee was full. However there was limitation of dorsiflexion on his right ankle at 0 degree, while plantar flexion is full. Despite all this, his gait is normal and he is able to fully squat normally. Anteroposterior and lateral radiographs of the leg showed malunited fractures of the midshaft of the right tibia and fibula with anterior angulation (Figure 2A \& 2B).

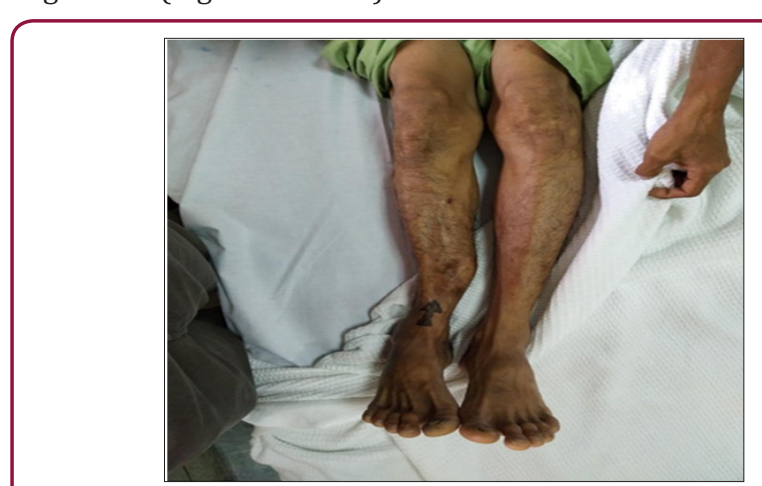

Figure 1A: Anteroposterior view of both leg.

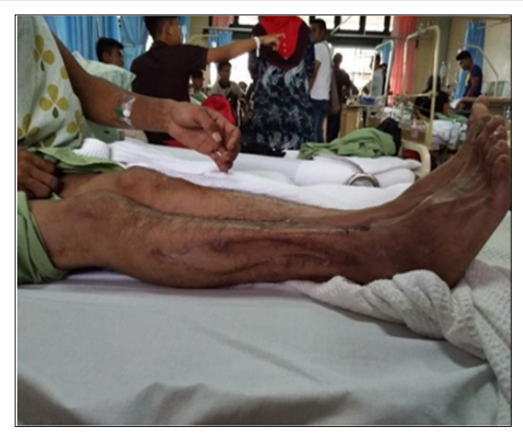

Figure 1B: Lateral view of leg. 


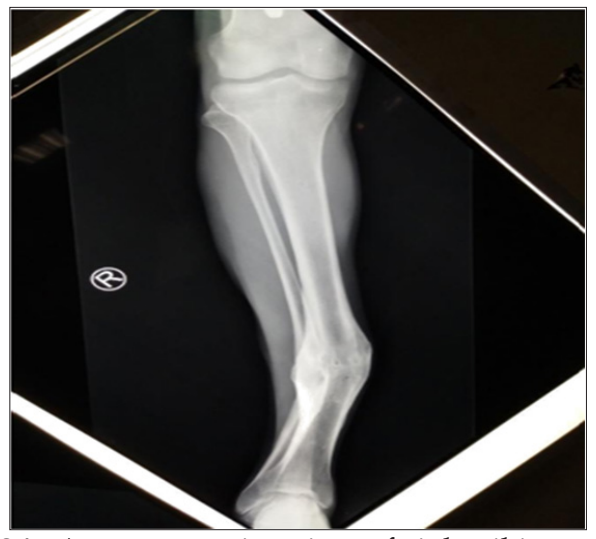

Figure 2A: Anteroposterior view of right tibia and fibula radiograph.

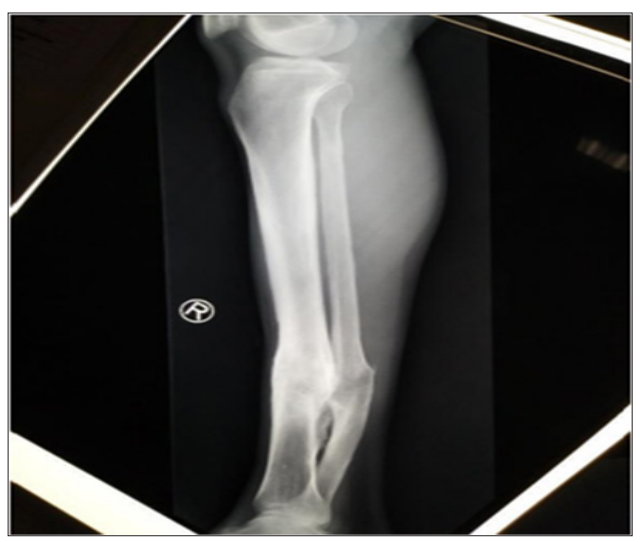

Figure 2B: Lateral view of right tibia and fibula radiograph.

\section{Preoperative Planning For Correction of Malalignment}

Angular deformity in frontal and sagittal plane was determined by standing face forward and lateral views, of the plain AP and lateral views radiographs of the tibia.

\section{Surgical Procedures}

The patient had a one stage operation to correct the deformities. The operation comprised fibula osteotomy through a lateral longitudinal incision over the fibula, chipping osteotomy at the tibia and osteoclasis of the tibial malunion through small antero-lateral approach and anteromedial approach to the CORA (Figure 2A). The intraoperative findings were malunited tibia fracture with thick posterior and medial cortex, $2 \mathrm{~cm}$ overlap of the fragments and medial angulation, as well as a cross union between the mid fibula and the tibia at the angulation site. The Achilles tendon and the posterior soft tissue of the distal leg were markedly contracted and shortened. The lengthening of the Achilles tendon was not done as patient did not give consent for that procedure to be done at the same setting (Figure 2B). At two month during follow-up period, he had a superficial wound infection where there is minimal serous discharge at the anterior tibia wound (Figures 3-9). Intraoperatively the infection looks superficial with low grade infection (Figure 10A). We decided to retain the implant with suppressive antibiotic with cloxacill in and ciprofloxacin after the deep tissue culture grew staphylococcus aureus and pseudomonas aeruginosa. The infection was controlled after administration of antibiotic. His fracture healed after 5 month and he was able to continue his work as a labourer without limitation. However he has occasional wound problem due to the prominent bone from the osteotomy site (Figures 10B \& 10C).

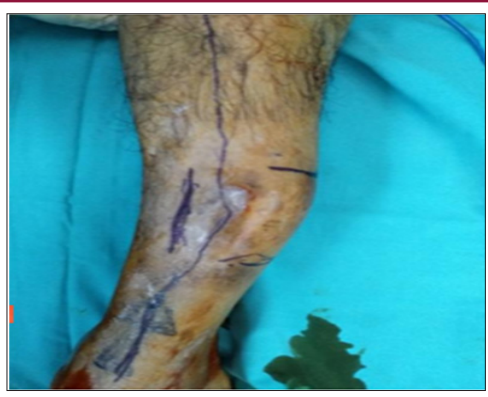

Figure 3: Step 1: Marking of the CORA and the intended incision for osteotomy.

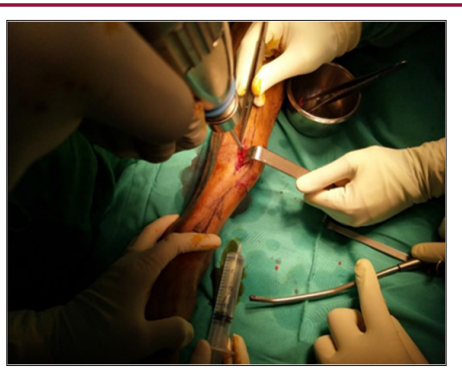

Figure 4: Step 2: osteotomy by using drill size $4.5 \mathrm{~mm}$.

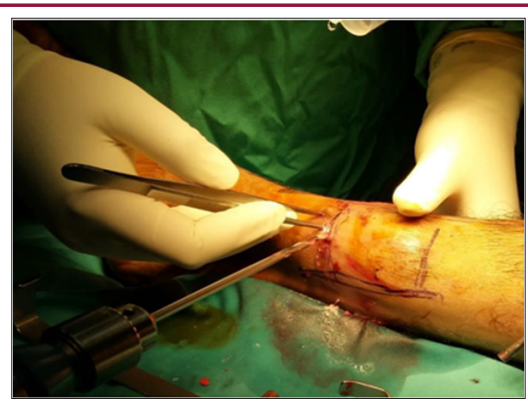

Figure 5: Step 3: Osteotomy by using drill size $4.5 \mathrm{~mm}$.

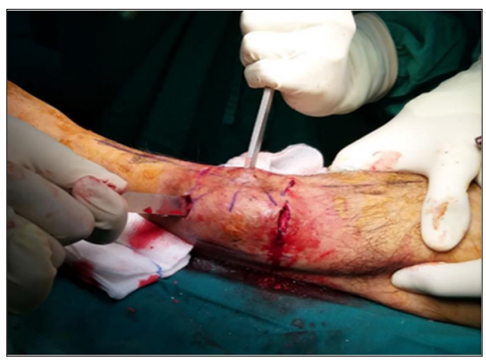

Figure 6: Step 4: Chipping osteotomy by using sharp osteotome.

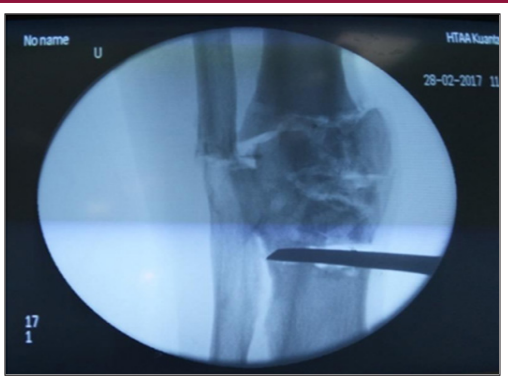

Figure 7: Check under image intensifier. 


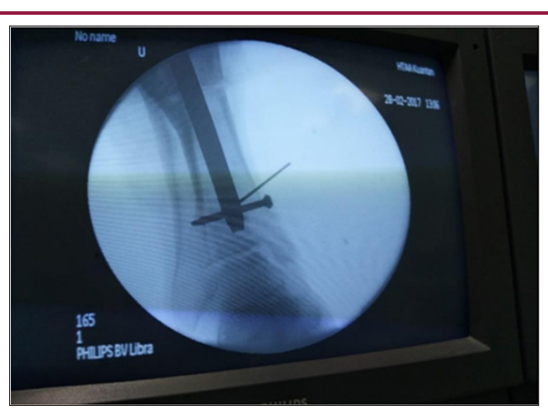

Figure 8: Interlocking nail was inserted. A K-wire was used to make sure the distal nail position and tibia angulation was in correct position before the distal screw was inserted.

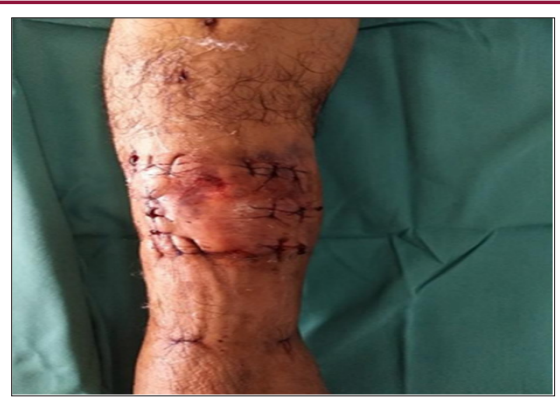

Figure 9: Wound after skin closure.

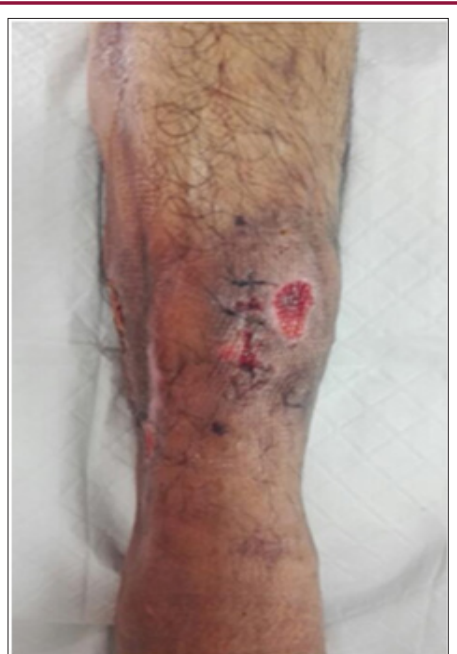

Figure 10A: Wound two month after debridement.

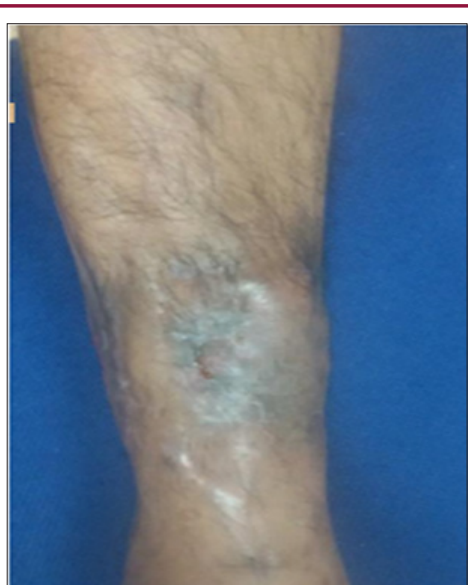

Figure 10B: Wound at 4 month during follow up.

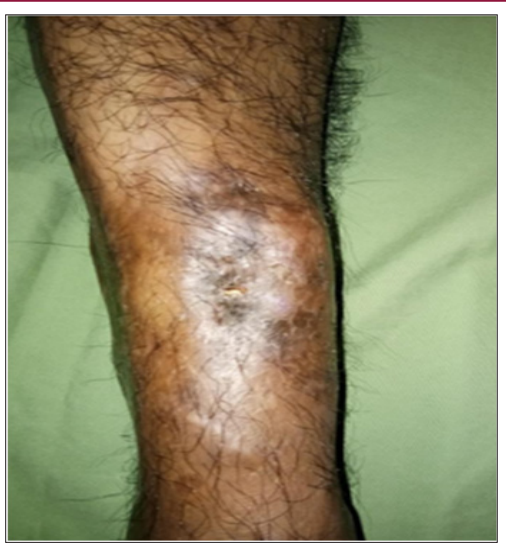

Figure 10C: Wound at 9 month.

\section{Discussion}

Long bone malunion can lead to chronic pain, contractures, early osteoarthritis and esthetic complaints. Standard surgical treatment relies on opening or closing wedge osteotomies, rotational osteotomies or combinations thereof. Osteotomy of long bones to correct angular deformity is a time-honoured procedure. It can be a straightforward operation, but one of the principal complications is the secondary displacement of the bone fragments. There are two basic osteotomy types for angular deformity correction:

a. Angulation-only osteotomies and

b. Angulation with translation osteotomies.

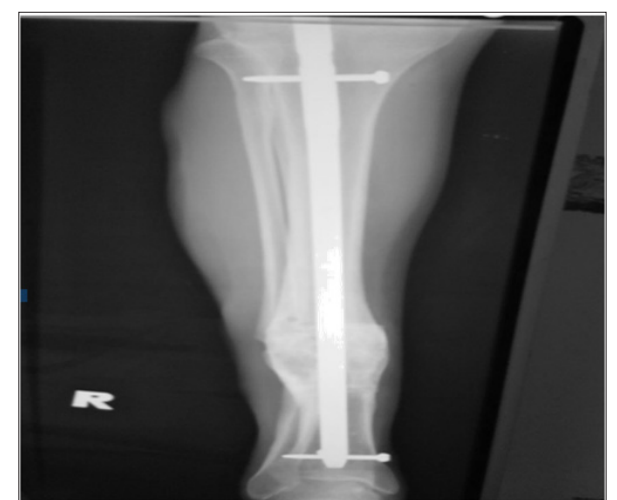

Figure 11A: AP radiograph at 9 months after surgery.

Angulation only osteotomies may be opening wedge or closing wedge types. Angulation with translation osteotomies may be straight cut or circular cut (dome). A disadvantage of the openwedge osteotomy is the resulting gap may prolong healing time; if the gap is filled with an avascular bone graft, it may increase the risk of nonunion and reoperation (Figure 11A \& 11B). The closed- wedge osteotomy, on the other hand, produces bone shortening. With multiplanar malunion, there is malalignment in the coronal, sagittal and transverse planes. Performing the correct osteotomy and the subsequent reduction and fixation is challenging Blankevoort et al. [1]. The Ilizarov technique is a satisfactory form of external fixation, especially in deformity correction. However, this method of treatment is very uncomfortable for patients; additionally, it presents certain inherent complications associated with external fixation, including pin track infection; joint infection; refracture; higher rates of delayed union, nonunion, and malunion; 
pin breakage; tendon transfixation; and vascular and nerve injury Kurtulmus [2].

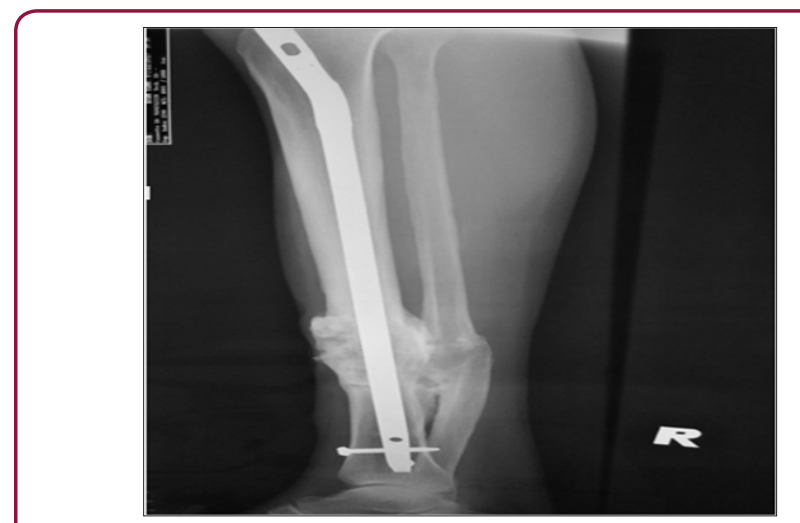

Figure 11B: Lateral radiograph 9 months after surgery.

A comparable common complication of tibia fracture is malunion, which is defined as a deviation from the normal anatomic configuration following healing of a fracture. This implies shortening, angular malalignment or rotational deformities. For long bone fractures, treatment goals include timely healing, restoration of normal anatomy and functional rehabilitation of patient. As for our note, the degree of acceptable deformity for tibia angulation noted by various authors is extremely variable. Rosemeyer and Pförringer suggested that surgery is indicated for valgus deformity of more than $12^{\circ}$, varus deformity of $>6$. Patil et al. [3]. Mal-union of the midshaft tibia due to fractures presents the patient with problem of appearance and function. It not only cause problem to the patient, but also to the managing doctor especially in the presence of a limited soft tissue envelop at the shaft of tibia. Fracture not only causing deep soft tissue scarring but it also causing contracture to the Tendo Achilles complex. This is evidence in our patient as he had developed callosity at the ball of the foot as a result of long standing equinus position of the ankle due to contracted gastroc-soleus complex (see pisture attached). Not only these fractures are comparatively common, it is usually troublesome to treat as the soft tissue envelope at the anteromedial surface of the tibia is thin and it usually causes severe bone and soft tissue injury.

Advances in operative treatment have provided the some required answer to the bony and soft tissue challenges of post traumatic lower limb deformities. Surgical choices that can be taken are immediate correction by surgery and fixation usually by distraction histiogenesis via external fixation with corticotomy to attain progressive correction. In some cases, amputation also ought to be as a mode of treatment if patient come with severe deformity and significant co-morbidities Dim et al. [4]. Chipping osteotomy was previously done on femur non-union and reported as a new technique to reconstruct long bone non-unions with shortening and/or malalignment by "chipping" without the use of bone graft. It is reported that with this technique, the union rate was almost the same as that obtained using other methods requiring bone grafting Watanabe \& Matsushita [5]. At the moment of writing, no data exists concerning the potential treatment of midshaft tibia malunion by using the "chipping" procedure as a method to achieved acute deformity correction. The treatment of tibia mal-union using the chipping osteotomy technique together with intramedullary device allowed us to maintain a biological stimulus to the bone and restoration of the mechanical alignment and thus providing optimum conditions for fracture healing without the need of bone grafting. However, due to the thin nature or the soft tissue envelope on the tibia, risk of wound breakdown and superficial infection need to be consider. This surgical technique should be considered as one option for the treatment of tibial shaft mal-union.

\section{References}

1. Blankevoort L, Streekstra GJ, Dobbe JGG, Pre KJ, Kloen P (2017) Computer-assisted oblique single-cut rotation osteotomy to reduce a multidirectional tibia deformity : case report. Strategies in Trauma and Limb Reconstruction 12(2): 115-120.

2. Kurtulmus T (2016) A novel intramedullary nail for use in the treatment of supramalleolar malunion and nonunion: A preliminary report of three cases. Acta Orthopaedica et Traumatologica Turcica 50(5): 6-11.

3. Patil VS, Kakani RR, Shaikh IU, Sagane SS, Bartakke GD (2017) Functional Outcome of Various Tibial Deformities following Intramedullary Nailing of Tibial Shaft Fractures : A Prospective Study 3(1): 1-8.

4. Dim EM, Itie JC, Inyang UC, Nwashindi A, Ugbeye ME, et al. (2015) CA SE REP ORT Post traumatic anterior angulation of the distal tibia following malunion: A case report of management in a resource constrained setting. Asian Journal of Medical Sciences 6(1): 108-111.

5. Watanabe Y, Matsushita T (2016) Femoral non-union with malalignment : reconstruction and biological stimulation with the chipping technique. Injury $47(6): 47-52$.

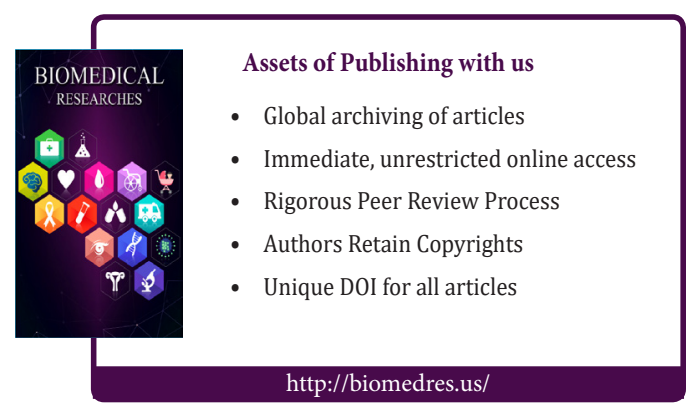

